\title{
Présentation : l'approche husserlienne de Dieu, entre métaphysique phénoménologique et phénoménologie de la religion
}

\section{Claudia Serban}

\section{CpenEdition}

Journals

Édition électronique

URL : https://journals.openedition.org/alter/2204

DOI : $10.4000 /$ alter.2204

ISSN : 2558-7927

Éditeur :

Association ALTER, Archives Husserl (CNRS-UMR 8547)

\section{Édition imprimée}

Date de publication : 1 novembre 2020

Pagination : 279-296

ISBN : 978-2-9550449-6-4

ISSN : 1249-8947

Référence électronique

Claudia Serban, «Présentation : I'approche husserlienne de Dieu, entre métaphysique phénoménologique et phénoménologie de la religion », Alter [En ligne], 28 | 2020, mis en ligne le 22 décembre 2020, consulté le 12 décembre 2021. URL : http://journals.openedition.org/alter/2204 ; DOI : https://doi.org/10.4000/alter.2204

Ce document a été généré automatiquement le 12 décembre 2021.

Revue Alter 


\title{
Présentation : l'approche husserlienne de Dieu, entre métaphysique phénoménologique et phénoménologie de la religion
}

\author{
Claudia Serban
}

\section{Les voies d'un « chemin a-religieux vers la religion »}

1 La publication, en 2014, du tome XLII des Husserliana, dont la troisième section est intitulée "Metaphysik: Monadologie, Teleologie und philosophische Theologie», a rendu accessible un nombre conséquent de textes où s'illustre l'approche husserlienne de la question de Dieu. Sans attendre ce travail d'édition, une littérature importante était toutefois déjà disponible sur le sujet, même en langue française. De la traduction, par Jacques Ridé, Alexandre Lowit et Henri Colombié, de l'article d'Alwin Diemer, «La phénoménologie de Husserl comme métaphysique ", publiée en 1954 dans Les études philosophiques ( $\mathrm{n}^{\circ} 1$, p. 21-49), à la contribution incisive de Jocelyn Benoist : «Husserl : au-delà de l'onto-théologie?", dans la même revue en 1991 ( ${ }^{\circ}$ 4, p. 433-458) et aux travaux plus récents et incontournables d'Emmanuel Housset (l'article « Husserl et le Dieu d'Aristote ", Les études philosophiques, n 4/1995, p. 481-496, et surtout, l'ouvrage Husserl et l'idée de Dieu, qui a vu le jour aux éditions du Cerf en 2010), la recherche husserlienne francophone a eu en outre le mérite de mener un effort précieux de traduction d'extraits clés qui a permis de fournir quelques premiers aperçus marquants de textes inédits ou difficilement accessibles.

2 Mais l'admirable travail mené par Thomas Vongehr et Rochus Sowa, les éditeurs du tome XLII des Husserliana, n'a pas rendu caduc pour autant le constat que les commentateurs de Husserl ont toujours fait au sujet de ses textes dits « théologiques »: comme le souligne Klaus Held, «Husserl n'a rien écrit de systématique sur ce thème ; il a abordé les questions relatives à la théologie métaphysique et à la philosophie de la 
religion seulement dans le cadre d'autres interrogations directrices $»^{1}$. Cet état d'éparpillement des sources a même pu alimenter l'impression d'une faible teneur philosophique de ces textes, et a rendu dans tous les cas urgente la question de leur "statut phénoménologique »².

3 Cette question est d'autant plus importante que Husserl n'a eu de cesse d'affirmer par ailleurs l'athéisme méthodologique de sa phénoménologie. L'une des expressions les plus nettes de cette revendication se trouve dans la Lettre à Przywara du 15 juillet 1932, traduite ici par Dominique Pradelle, où le phénoménologue met en avant sa «manière athéologique de philosopher (atheologisches Philosophieren)»(Briefwechsel, Husserliana Dokumente III/7, p. 237). Loin d'être isolé et occasionnel, cet aveu est corroboré par bien d'autres qui donnent à lire quelques variations sur ce même thème. Ainsi, fin 1935, Husserl écrit de manière éloquente à son ami Gustav Albrecht: "Pour moi, la philosophie est en dernière instance mon chemin a-religieux vers la religion, ou pour ainsi dire mon chemin athée vers Dieu " (Briefwechsel, Husserliana Dokumente III/9, p. $124)^{3}$. Cette dernière formulation aide à comprendre que l'athéisme méthodologique de Husserl ne consiste pas tant dans une mise entre parenthèses intégrale de la question de Dieu que dans la décision de l'aborder sans aucun recours à la foi ou à la Révélation, uniquement selon les lignes directrices de la méthode phénoménologique. En ce sens, un important manuscrit de travail de 1933 (A VII 9, « Horizont») publié au tome XXXIX des Husserliana fournit une définition importante : « une science qui ne présuppose pas la révélation [...] est athée. Par conséquent, si une telle science menait tout de même à Dieu, son chemin du divin serait un chemin athée vers Dieu (wäre ihr Gottesweg ein atheistischer Weg zu Gott)» (Hua XXXIX, p. 167). Et dans des termes qui se laissent sans doute appliquer à sa propre phénoménologie, Husserl affirme dans un texte datant probablement de 1934 et publié désormais au tome XLII des Husserliana: «Une philosophie autonome [...] aboutit nécessairement à une téléologie et une théologie philosophiques - en tant que chemin non confessionnel vers Dieu» (Hua XLII, p. 259) $»^{4}$.

4 Cette profession de foi paradoxale - celle d'un athéisme qui a tout de même le sens d'une quête de Dieu - se retrouve également, dans un registre plus personnel et existentiel, dans les Conversations avec la sœur Adelgundis Jaegerschmid, traduites ici par Julien Farges, qui attribuent à Husserl le propos suivant :

La vie humaine n'est rien d'autre que le chemin vers Dieu. J'essaie d'atteindre ce but sans preuve, sans méthode et sans aide théologiques, c'est-à-dire d'atteindre Dieu sans Dieu. Je dois en quelque sorte éliminer Dieu de mon existence scientifique afin de baliser la voie qui mène à Dieu pour les gens qui, contrairement à vous, n'ont pas l'assurance de la foi grâce à l'Église. Je sais que ma façon de procéder pourrait être dangereuse pour moi si je n'étais pas un homme profondément lié à Dieu et croyant au Christ ${ }^{5}$.

5 Devient-il dès lors à nouveau difficile de dissocier, chez Husserl, la foi personnelle et la recherche philosophique? Sans le concéder trop rapidement, nous pouvons tout de même remarquer, en suivant le constat de Thomas Vongehr et de Rochus Sowa dans leur précieuse Introduction au tome XLII des Husserliana, qu'indéniablement, « dans sa riche correspondance, scientifique comme privée, Husserl s'est exprimé plusieurs fois au sujet de ses convictions religieuses ", et que, grâce à ces nombreux témoignages, les textes (publiés ou inédits) où sa métaphysique phénoménologique s'annonce ou amorce son déploiement - le plus souvent sous la forme d'une téléologie aux implications 
théologiques - "perdent le caractère d'une pure spéculation» et dévoilent leur « sérieux existentiel (existenziellen Ernst)» (Hua XLII, p. lxxix).

Il convient cependant de préciser que les textes «métaphysiques » ou " téléologiques » publiés au tome XLII des Husserliana mettent en relief seulement l'une des voies par lesquelles la phénoménologie de Husserl tâche d'« atteindre Dieu sans Dieu » - c'est-àdire, sans le concours de la foi ou de la Révélation -, alors qu'il s'agit en réalité d'un cheminement complexe et multiple. Tout d'abord, contrairement à l'impression que ce geste hasardé n'est effectué par Husserl que dans des manuscrits de recherche, il faut rappeler qu'une voie épistémologique (1) vers Dieu - défini alors classiquement en tant que « représentant idéal de la connaissance absolue » (Hua III/1, p. 351) - est tracée dès le premier tome des Idées directrices ${ }^{6}$, et perdure jusque dans la conférence de 1935 sur La crise de l'humanité européenne, où il est question même d'une «logification » de Dieu au terme d'un " processus d'idéalisation » qui en fait significativement le "porteur du logos absolu» (Hua VI, p. 335 ; trad. par G. Granel, p. 370). La voie téléologique (2), particulièrement mise à l'honneur au tome XLII des Husserliana, est sans doute double, pour autant qu'elle s'amorce à la fois sur le terrain de la téléologie mondaine (Weltteleologie, Hua Mat IX, p. 188 et passim; Hua XLII, p. 210 et passim) et sur celui de la téléologie de la raison (la Vernunftteleologie présente dans la Krisis et dans les textes connexes contemporains - désignée aussi parfois comme "téléologie subjective (subjektive Teleologie)", Hua Mat IX, p. 203). La voie de la téléologie rationnelle ou subjective recoupe dans une certaine mesure ce qu'on pourrait appeler la voie de l'immanence ou, mieux encore (pour désactiver la résonance augustinienne de la référence à l'intériorité)), la voie de la monadologie (3), par laquelle le «chemin non confessionnel vers Dieu» s'enracine dans l'immanence de l'ego transcendantal. Vient ensuite la voie de l'éthique (4), qui fait qu'à la «logification » de Dieu fait pendant son «éthicisation » (l'Ethisierung Gottes dont fait mention un texte de 1924, Hua XLII, p. 255, n. 1). Comme la voie épistémologique, cette voie éthique a indéniablement une certaine résonance kantienne ${ }^{8}$, car l'éthicisation de Dieu est indissociable de « la tâche infinie de l'éthicisation de l'humanité »(Hua XLII, p. 332) ${ }^{9}$. Et pour que cet inventaire puisse prétendre à une certaine complétude ${ }^{10}$, il faut mentionner aussi la voie doxique (5), qui approche Dieu comme objet de foi et la foi religieuse à travers l'angle de la croyance, ainsi que la voie de la culture et des institutions (6), qui s'esquisse dans les textes où Husserl se réfère aux religions instituées et à leurs pratiques communautaires.

7 Toutes ces voies - qui ne manquent certes pas de se croiser et de s'intriquer - menant à Dieu sans Dieu trouvent leur point de départ dans l'immanence phénoménologique délimitée par la pratique de la réduction : elles restent donc radicalement distinctes de la voie classique (celle de la métaphysique traditionnelle et des approches qui la prolongent) qui approche Dieu en démontrant son existence et en s'inclinant devant sa transcendance. C'est pourquoi Husserl peut légitimement défendre sa démarche contre toute accusation de rechute dans une spéculation métaphysique ou religieuse, comme il le fait dans un texte de 1910: "Nous ne faisons pas de mysticisme ici (Wir treiben hier keine Mystik)»(Hua XLII, p. 158) ${ }^{11}$. Et dans la lettre qu'il écrivit à Rudolf Otto le 5 mars 1919, il met précisément en garde contre le danger qu'en matière de philosophie de la religion, «le métaphysicien (le théologien)» emporte «sur ses ailes le phénoménologue ", qui procède alors comme "l'ange qui voile le regard de ses ailes ${ }^{12}$. Le parcours non confessionnel vers Dieu tenté par Husserl veut rester en revanche, d'un bout à l'autre, un parcours immanent - situé dans un horizon égologique et non 
théologique -, ce pourquoi Emmanuel Housset pouvait considérer que, chez Husserl, "le chemin de l'homme vers Dieu demeure dans une démarche athée un chemin de l'homme vers lui-même » (op. cit., p. 158).

\section{II. « D'en bas » « vers le haut » : le niveau suprême de la progression phénoménologique}

8 La question demeure pourtant de savoir quelle place l'on peut attribuer, au sein du corpus husserlien, à ce champ thématique que l'on peut regrouper à la fois - sans que cela revienne entièrement au même - autour de la question de Dieu et autour des "problèmes éthico-religieux" dont Husserl fait souvent mention. Alors que dans la Lettre à Parl Welch de juin 1933, que nous avons traduite ici ${ }^{13}$ avec Natalie Depraz, Husserl refuse fermement de parler de sa philosophie en termes de système, le Nachlass montre qu'au tout début des années trente, au moment où il laisse de côté la réécriture des Méditations cartésiennes, il avait bel et bien envisagé le projet d'une exposition systématique de sa phénoménologie. Dans leur Introduction au tome XLII des Husserliana, Thomas Vongehr et Rochus Sowa reprennent l'indication d'un tel projet publiée par Iso Kern en 1973, dans son Introduction au tome XV contenant les textes tardifs sur l'intersubjectivité. L'exposé systématique de la phénoménologie transcendantale comprendrait, d'après cette esquisse que Husserl en a donnée en 1930, cinq tomes, dont le dernier rassemblerait les intitulés suivants: "Genèse transcendantale du monde objectif. Genèse transcendantale des êtres humains et des humanités. Les problèmes de la génération (Die Generationsprobleme). Les problèmes de la conservation de soi (Selbsterhaltung), de l'être humain dans l'authenticité (in der Echtheit). Humanité et destin. Les problèmes téléologiques et les problèmes relatifs à Dieu » (Hua XLII, p. Xxiii ; Hua XV, p. Xxxvi). Si la méthode phénoménologique prônée par Husserl a toujours consisté à partir "d'en bas (von unten) », elle n'entendait donc pas reculer pour autant, une fois ce point de départ assuré et maintenu, à s'avancer résolument " vers le haut ». Selon cette progression, de manière tout aussi significative, la mention passagère de la fin des Méditations cartésiennes, faisant état des " problèmes éthico-religieux » (Hua I, p. 182 ; trad. par M. de Launay, p. 208), devient l'index d'un ensemble thématique qui non seulement est censé couronner le projet systématique de la phénoménologie, mais peut aussi acquérir à présent une consistance supplémentaire grâce aux manuscrits de recherche désormais édités en grande partie.

9 Cet étagement de la philosophie phénoménologique, qui place les problèmes téléologiques, théologiques et éthico-religieux au sommet de son édifice, est suggéré par Husserl à de nombreuses reprises. Ainsi, en 1932, il écrit à son ami Gustav Albrecht que :

[...] parmi toutes les questions, celles qui sont situées le plus haut [...] sont les questions métaphysiques; elles concernent la naissance et la mort, l'être ultime du Je et du Nous objectivé en tant qu'humanité, ou encore la téléologie, qui nous conduit en dernière instance à la subjectivité transcendantale et à son historicité transcendantale ; et naturellement aussi la question suprême : l'être de Dieu en tant que fondation ultime d'une telle téléologie [...] (Briefwechsel, Husserliana Dokumente III/9, p. 84)

10 Et en dehors de la Correspondance de Husserl, une Conversation avec Dorion Cairns, datée du 24 novembre 1931, renchérit fermement : «Les questions éthico-religieuses sont les ultimes questions de la constitution phénoménologique » (Conversations with 
Husserl and Fink, p. 46-47 ; trad. par J.-M. Mouillie, p. 134). Ces assertions confirment que l'annonce de la fin des Méditations cartésiennes n'avait pas vocation à désigner un champ problématique (métaphysique) extérieur à la phénoménologie, mais un niveau supérieur de développement (correspondant à ce que serait une métaphysique phénoménologique) qui n'avait pas encore été atteint. Or cela ne veut pas dire pour autant que la phénoménologie transcendantale chemine vers Dieu en suivant une progression prescrite au bout du compte par la métaphysique traditionnelle. Situer la question de Dieu parmi " les ultimes questions de la constitution phénoménologique ", c'est bien plutôt en modifier radicalement l'approche, comme le suggère le même propos rapporté par Dorion Cairns: "Dieu en tant que principe du bien demeure essentiellement mondain. C'est seulement quand la nature de la conscience transcendantale est comprise que la transcendance de Dieu peut l'être ${ }^{14}$ (Conversations with Husserl and Fink, p. 46-47 ; trad. par J.-M. Mouillie, p. 134).

11 En esquissant le même type de progression "vers le haut ", Husserl affirme en toute clarté dans la lettre à Welch ici traduite que «le couronnement suprême de la problématique de la philosophie phénoménologique est la question du "principe" de la téléologie " (Briefwechsel, Husserliana Dokumente III/6, p. 461). À l'appui de cette centralité cruciale de la question téléologico-théologique, Thomas Vongehr et Rochus Sowa citent d'autres mentions convergentes, comme celle, fort précieuse, d'une lettre au Père Feuling de mars 1933 où Husserl présente la « question de Dieu » comme étant «la question suprême et ultime (höchste und letzte Frage) dans l'architecture systématique (Systembau) de la méthode phénoménologique », pour préciser aussitôt, dans des termes parfois proches de ceux dans lesquels il s'adressera à Welch quelques mois plus tard:

Je suis assez reconnaissant d'avoir pu configurer et développer explicitement la méthode jusqu'à apercevoir le lieu théorique du problème en tant que phénoménologique (um den theoretischen Ort des Problemsals eines phänomenologischen zu sehen): d'abord, comme celui du pro-blème de la possibilité de la totalité transcendantale (la téléologie et son principe : le "sur-étant" [...].

12 Husserl précise toutefois aussitôt, pour éviter tout risque de mécompréhension : " Mais je n'ai pas de théologie, fût-ce même en un sens tout à fait général (Aber eine Theologie, sei es auch eine ganz allgemeine, habe ich nicht) $»^{15}$ (Briefwechsel, Husserliana Dokumente III/ 7, p. 87-88). Il ne faut donc pas se précipiter à séparer, chez Husserl, les implications à allure théologique des prémisses qui sont les leurs, ou à renverser l'ordre des raisons et celui de la progression méthodique de la phénoménologie : la téléologie et son ancrage égologique précédent et conditionnent nécessairement leur corollaire théologique.

Le champ des "problèmes éthico-religieux» est intimement lié à la question téléologico-théologique. Bien que, selon la même lettre à Welch, il s'agisse aussi, plus généralement des «questions de la vie éthique, de la vie dans l'humanité authentique et inauthentique, dans la satisfaction ou dans l'infélicité ultimes (de l'harmonie ou la disharmonie individuelles ou sociales) ", ces questions se laissent identifier en dernière instance à « la problématique omni-englobante qu'on peut aussi désigner comme étant celle de la téléologie universelle » (Briefwechsel, Husserliana Dokumente III/6, p. 461). Et il appert clairement que ce domaine n'est pas extérieur à la phénoménologie, mais relève d'un niveau d'analyse supérieur dont l'accès nécessite une application particulière :

[...] les problèmes éthico-religieux sont des problèmes de cette sorte relevant du niveau suprême. (En tant que problèmes scientifiques, ils ne peuvent donc pas être conquis à peu de frais comme le voulait l'ontologisme fondamentalement naïf de 
Scheler.) C'est précisément pourquoi j'ai gardé le silence dans mes écrits au sujet des problèmes de philosophie de la religion. Et pourtant, dans ceux-ci et dans les publications à venir, des chemins sont déjà préparés pour poursuivre le travail en ce sens et pour formuler les véritables problèmes de philosophie de la religion. (Briefwechsel, Husserliana Dokumente III/6, p. 459) ${ }^{16}$

14 Ce passage important de la lettre à Welch confirme tout d'abord le sens de la progression phénoménologique: partir «d'en bas», c'est aussi préparer méthodiquement le terrain pour l'approche du niveau supérieur, donc assurer le bienfondé de la progression "vers le haut ». La question religieuse requiert, aux yeux de Husserl, de longs et patients préparatifs pour pouvoir être abordée et élaborée d'une manière rigoureuse, donc proprement philosophique. Son approche systématique, différée jusque dans les années trente et finalement restée en chantier, exigeait à ses yeux que la phénoménologie ait atteint sa pleine maturité et ait parcouru et clarifié les niveaux préalables sur lesquels s'édifient l'expérience et la conscience religieuse.

Tâchons de préciser davantage l'articulation interne de cette progression «vers le haut » ou du chemin non confessionnel vers Dieu suivi par la phénoménologie de Husserl, en nous interrogeant sur la manière dont l'égologie transcendantale appelle un prolongement ou un couronnement qui prend des allures théologiques. Nous pouvons, pour commencer, souligner avec Emmanuel Housset que «c'est en approfondissant sa compréhension de la vie transcendantale que Husserl en vient à parler de Dieu» (op. cit., p. 166). Mais Dieu n'est pas (comme chez Augustin ou Descartes, que Husserl cite à des endroits décisifs, ou comme plus tard chez Levinas) le transcendant (tout-puissant, infini ou incompréhensible) qui fait éclater le cadre de l'immanence égologique: ce sont, au contraire, l'absolutisation et l'idéalisation des attributs égologiques qui le font surgir furtivement, comme au $\S 49$ des Ideen I, lorsqu'en reprenant des termes cartésiens, il est dit de " l'être immanent » de l'ego qu'il n'a « besoin d'aucune autre chose pour exister (nulla re indiget ad existendum) » (Hua III/ 1, p. 92 ; trad. par J.-F. Lavigne, p. 150). Dans l'horizon ouvert par le tournant transcendantal, cette question du statut ontologique de l'ego et de son absoluité est indissociable par ailleurs de ses enjeux épistémologiques, et Dieu s'y profile alors comme le «représentant de la connaissance absolue ». Mais avant que l'univocité gnoséologique entre l'ego et Dieu soit acquise dans les Ideen I, une « conscience divine omni-englobante (göttliches Allbewusstsein) $»^{17}$ semble appelée à surmonter la finitude de la conscience de l'ego humain, dans des textes comme le Complément IV du tome XIII des Husserliana, datant de 1908. La phénoménologie transcendantale transpose ainsi diversement, dès ses premières élaborations, la dualité traditionnelle de la connaissance finie et de la connaissance infinie, sans que Dieu recouvre pour autant autre chose qu'une auto-idéalisation de l'ego.

Mais même indépendamment de ces considérations épistémologiques, la monadologie sui generis par laquelle s'exprime la conception husserlienne de la subjectivité et de la communauté transcendantales paraît avoir été vouée dès le départ à tracer l'un des plus nets "chemins non confessionnels vers Dieu». Les textes édités dans la section "Metaphysik: Monadologie, Teleologie und philosophische Theologie» du tome XLII des Husserliana montrent en effet que c'est très tôt (encore une fois, dès 1908) qu'elle a amorcé son propre prolongement sous la forme d'une métaphysique phénoménologique ${ }^{18}$ qui ne manque pas de croiser la question de Dieu. Il s'impose donc de reconnaître qu'il ne s'agit ni d'un ajout tardif, ni d'un complément facultatif de la pensée husserlienne de l'ego ou de la vie monadique. Bien que relevant (comme nous 
l'avons amplement souligné) d'un niveau supérieur de la problématique générale de la constitution, la question métaphysique de la téléologie et de son principe théologique est aperçue et soulevée par Husserl dès les premières formulations de sa phénoménologie transcendantale, même si, à ce stade, elle est souvent énoncée de manière programmatique et son élaboration se trouve sans cesse différée. Les textes publiés ne font pas exception à cet égard, comme le montre, dans les Ideen I de 1913, la Remarque qui accompagne le $\S 51$ et qui pose «l'immanence de Dieu dans la conscience absolue " comme corollaire du fait que le "principe théologique» de la "téléologie immanente » qui régit « la factualité contingente » ne saurait être « un Dieu mondain », ou " une transcendance selon le monde ", ni un principe y exerçant une action causale ${ }^{19}$ (Hua III/1, ; trad. par J.-F. Lavigne, p. 157). Le $\$ 58$ du même ouvrage accomplit ensuite fameusement la «mise hors circuit» de la «transcendance de Dieu», après que ce dernier a été à nouveau appelé par la "merveilleuse téléologie » qui empêche que la factualité de tout ce qui existe puisse être tenue pour synonyme d'irrationalité ou de désordre. Cependant, encore une fois, cette mise hors circuit ne conduit pas à rompre avec toute pensée de Dieu : elle ne concerne que sa transcendance, ce qui exprime la décision de refuser de l'aborder en quittant l'immanence de l'ego ou l'espace de sens délimité par la réduction phénoménologique.

L'œuvre publiée de Husserl n'a donc pas entièrement passé sous silence la question téléologico-théologique, bien que la progression «d'en bas » "vers le haut» de la démarche phénoménologique ait le plus souvent exigé d'en repousser le traitement. La Crise des sciences européennes mentionne elle aussi, au $\S 3$, dans une optique plus historique, la «téléologie et son [...] plus haut principe : Dieu » (Hua VI, p. 5 ; trad. par G. Granel, p. 12), apportant une nouvelle confirmation du fait que Husserl n'a pas abordé ces questions exclusivement dans les manuscrits de recherche restés inédits de son vivant. À lire cette page de la Krisis, l'on pourrait même avoir l'impression qu'il attribue ouvertement à la phénoménologie transcendantale la tâche de réinvestir les questions métaphysiques laissées de côté par la science positive :

Le problème de Dieu contient manifestement le problème de la raison «absolue » comme source téléologique de toute raison dans le monde, le problème du "sens " du monde. [...] Toutes ces questions métaphysiques au sens large, c'est-à-dire les questions spécifiquement philosophiques au sens habituel du terme, dépassent le Monde en tant qu'Universum des simples faits. Elles le dépassent précisément en tant que questions qui remuent (im Sinne haben) l'idée de la raison. (Hua VI, p. 7 ; trad. par G. Granel, p. 14)

18 Il est vrai toutefois que dans les manuscrits de travail, le discours de Husserl se laisse porter bien plus facilement par un certain pathos et dévoile, à l'arrière-fond de sa réflexion, un engagement personnel et existentiel que les textes publiés dissimulent sobrement. Ainsi peut-il écrire, à l'époque de la rédaction de la Krisis, que «Dieu parle en nous", dans un appendice à son projet de contribution au VIIIe Congrès international de philosophie de Prague (1934). De manière significative, l'apparition de la question de Dieu y est suscitée à nouveau par le problème de la téléologie :

La «téléologie » découvre que Dieu parle en nous, Dieu parle dans l'évidence des décisions qui, à travers tous les aspects du monde fini, indiquent l'infinité. Je suis sur une route. Où va cette route? Quelle est ma voie, la voie qui me conduit à l'infini, qui me garantit à tout moment que je suis sur la bonne voie et qui, à chaque bévue me montre que je suis intérieurement dans l'aveuglement et dans l'erreur, qui me permet de voir où j'agis selon ma vocation et où j'agis à l'encontre de ma vocation? En moi, toutes les bonnes routes conduisent à Dieu [... $]^{20}$ 
19 Dieu apparaît ici comme une exigence - l'exigence ultime - de la vie égologique. Cette thèse exprime sans doute la façon éminente dont se croisent les voies, illustrant le « chemin non confessionnel vers Dieu » pratiqué par la phénoménologie husserlienne, que nous avons appelées monadologique et éthique.

Soulignons aussi que n'est pas seulement l'inquiétude (évoquée au tout début de la lettre à Welch) du contexte historique des années trente qui suscite chez Husserl de telles interrogations et réponses. Le fait que le nom de Dieu exprime l'exigence suprême de la vie de l'ego et le fruit de son auto-idéalisation trouve déjà une illustration particulièrement éloquente dans un manuscrit de recherche datant probablement de 1925 et édité au tome XLII des Husserliana :

Pour parler en termes religieux: Si un Je doit pouvoir vivre de manière concordante, il doit y avoir une nature et Dieu doit l'avoir créée. L'être humain ne peut être satisfait que lorsqu'il peut vivre orienté vers l'idéal de soi-même en tant qu'être absolument parfait et réaliser celui-ci de manière active dans un effort infini. Il doit porter un Dieu en lui-même (Er muss einen Gott in sich tragen). (Hua XLII, p. 175)

21 La vie « concordante » de l'ego dont il est question dans ce passage n'a sans doute pas une acception de prime abord éthique, mais cette acception demeure fondamentale dans la mesure où, comme nous l'avons déjà suggéré, la cohésion et l'articulation interne des "problè-mes éthico-religieux » est décisive chez Husserl. Et puisque la question éthique concerne l'engagement personnel du phénoménologue, son discours peut s'autoriser un ton plus personnel, aux infléchissements existentiels, comme à la fin d'un texte de 1924 :

Je ne peux être heureux, je ne peux l'être dans toute la souffrance, le malheur, dans toute l'irrationalité de mon monde environnant que si je crois que Dieu existe et que ce monde est le monde de Dieu. Et si je veux m'en tenir au devoir absolu avec toute la force de mon âme [...], alors je dois croire absolument qu'il existe. La foi (Glaube) est l'exigence absolue et suprême. (Hua XLII, p. 203) ${ }^{21}$

La foi pratique en Dieu, elle aussi à forte résonance kantienne, précise d'une nouvelle manière l'agencement interne des "problèmes éthico-religieux $»^{22}$ et reflète le type d'engagement « doxique » qu'ils réclament.

Nombreuses sont ainsi les illustrations de la manière dont la phénoménologie, dans son ambition de pousser le plus loin possible la compréhension de la vie transcendantale, avance vers le sommet représenté par les questions éthiques, religieuses ou théologiques, mais sans quitter pour autant le domaine de l'égologie et le chemin de la réduction. Le texte qui reflète sans doute le mieux cette démarche singulière se trouve significativement, non dans un manuscrit de recherche ayant dû attendre longuement son édition, mais au $\S 99$ de Logique formelle et logique transcendantale (1929), dont l'intitulé («Subjectivité psychologique et subjectivité transcendantale. Le problème du psychologisme transcendantal») soulève un problème fondamental que la phénoménologie de Husserl n'a eu de cesse d'affronter sur une trentaine d'années. L'approche de ce problème donne l'occasion de poser avec fermeté et clarté le cadre on pourrait même dire, en reprenant l'expression de la lettre à Heuling, le «lieu théorique »- que la phénoménologie assigne à la question de Dieu :

L'a priori subjectif, c'est ce qui précède l'être de Dieu et du monde et tout ce qui, sans exception, existe pour moi qui pense. Dieu, lui aussi, est pour moi ce qu'il est, de par ma propre effectuation de conscience ; de cela, je ne peux pas détourner les yeux dans la crainte angoissée de ce qu'on peut penser être un blasphème, mais au contraire je dois voir le problème. Ici aussi, comme à l'égard de l'alter ego, 
" effectuation de conscience " ne veut pas dire que j'invente et que je fais cette

transcendance suprême. (Hua XVII, 222 ; trad. par S. Bachelard, p. 336) thèse. Le premier concerne la référence - fût-elle rare (sinon rarissime) sous la plume de Husserl - à la prière. Elle se trouve mise en avant dans le bref Appendice XXV du tome XLII des Husserliana, daté de 1931, que nous avons choisi de traduire ici. Thomas Vongehr et Rochus Sowa mentionnent et citent dans leur Introduction ce texte singulier, sans toutefois le commenter. Il appelle cependant plusieurs remarques qui sont à même de souligner son intérêt. Tout d'abord, il fournit sans doute quelques éléments de réponse à la question posée par Angela Ales Bello au sujet de la conversion au christianisme de Husserl et d'Edith Stein - celle de savoir « pourquoi Husserl choisit le christianisme luthérien et Stein le catholicisme romain » (op. cit., p. 72). La prière, telle que Husserl la comprend, se caractérise par son «attitude intérieure » qui l'éloigne du culte des images ${ }^{25}$ - tentatives foncièrement inadéquates de représenter Dieu dans l'extériorité -, et cette orientation intérieure de la prière est le corollaire de «l'être intérieur (Innesein)» de Dieu. La résonance frappante de cette expression ${ }^{26}$ semble corroborer l'aveu surprenant fait par Husserl à Dorion Cairns en juin 1932, lorsqu'il «déclara qu'il pouvait reprendre telles quelles des pages entières de Maître Eckhart » (Conversations with Husserl and Fink, p. 91 ; trad. par J.-M. Mouillie, p. 185) ${ }^{27}$.

qui est en revanche encore plus étonnant, c'est que la mise en évidence de l'orientation intérieure de la prière conduit à déceler quelque chose de « commun à la conscience religieuse et à l'instinct » : « l'orientation intentionnelle est là, mais avec un horizon vide qui n'est pas du tout formé, [elle est] <orientée vers> un but qui n'a aucune structure de familiarité préfigurée " (Hua XLII, p. 246). Serait-ce sous les espèces de l'horizon vide, ou du telos obscur, que Husserl pense (enfin) la transcendance propre à Dieu? Si c'est le cas, aux antipodes du remplissement et de la donation intuitive, la prière et l'attitude religieuse qu'elle manifeste semblent condamnées à ne jamais pouvoir parvenir à l'évidence.

C'est néanmoins de manière assez exceptionnelle que Husserl suggère un tel fonctionnement de l'expérience religieuse, induit par l'analogie singulière avec l'instinct. Il est en effet bien plus courant de trouver sous sa plume une référence à l'idée d'« évidence religieuse ». Ainsi, dans la lettre à Przywara de 1932 traduite ici par Dominique Pradelle, Husserl aborde l'évidence religieuse comme un cas particulier de l'évidence tout court, susceptible de faire l'objet d'une élucidation phénoménologique :

[...] ma méthode phénoménologique suffit à résoudre tous les véritables problèmes d'évidence (Evidenzproblemen), [et] en effectuant une clarification réflexive des 
effectuations constitutives qui s'y sont déposées, elle permet d'accéder à l'élucidation ultime de la teneur de sens et de la portée de ces évidences. Cela vaut aussi pour les évidences religieuses (religiöse Evidenzen). (Briefwechsel, Husserliana Dokumente III/7, p. 237) ${ }^{28}$

(pour reprendre les termes du fragment de 1931), si elle se laisse décrire à la fois en termes d'horizon vide et d'évidence originaire ? Il serait assurément tentant de recourir à la distinction entre prière (ou religion) authentique et inauthentique, où le critère de démarcation serait précisément la nature de l'évidence en question. En ce sens, selon un texte de 1930, "l'évidence religieuse au sens le plus authentique (eigentilch) » est " celle d'une religion "véritable (echt)", par contraste avec l'"évidence" des idoles relatives à la finitude (auf Endlichkeit bezogen)» (Hua XLII, p. 447-448). Mais même alors, il se pourrait que l'évidence originaire de la prière soit peu de chose par rapport à d'autres évidences se trouvant à la portée du phénoménologue. C'est ce que suggère lapidairement un passage des Conversations avec la sœur Adelgundis Jaegerschmid, traduites ici par Julien Farges : «Y a-t-il, dans la vie religieuse, évidence plus assurée et plus authentique que la prière ? [...] Et pourtant, la prière n'est pas une instance d'évidence ultime, absolue » (Conversation du 23 mars 1937). Toutes les évidences ne se situent donc pas sur le même plan et n'ont pas la même valeur - ce qui ne porte pas atteinte par ailleurs à leur légitimité. C'est à l'appui de cette interprétation que vient aussi la position exprimée par Husserl au sujet de la mystique, dans une Conversation avec Dorion Cairns de juin 1932 :

Husserl parla du mysticisme. Toute évidence authentique possède son droit. La question est toujours celle de la portée d'une évidence quelconque. Ceci s'applique aux évidences particulières que le mystique possède. [...] Il doute pourtant du caractère suffisant de la pratique du mysticisme. Le "réveil" qui suit l'expérience mystique doit probablement être bien pénible. En revanche, la prise en vue de la rationalité du monde que l'on gagne par la vraie recherche scientifique se manifeste à travers toute expérience future. La différence est en outre celle qui sépare le plaisir passif et le travail. Le mystique néglige le travail. Les deux sont nécessaires. (Conversations with Husserl and Fink, p. 91 ; trad. par J.-M. Mouillie, p. 185)

\section{Attitude religieuse et attitude phénoménologique : une affinité intime?}

Reste encore - et c'est l'autre intérêt du texte de 1931 que nous avons traduit - à préciser le sens du rapprochement avec la démarche phénoménologique sur lequel débouche la prise en compte de l'orientation intérieure que l'être intérieur de Dieu prescrit à la prière :

Cette orientation intérieure est parallèle à l'orientation intérieure phénoménologique dans laquelle, en passant par mon intériorité, un chemin va vers tous les autres (des autres intérieurs, et non pas des êtres humains extérieurs ayant une réalité spatio-temporelle) et par là seulement vers le monde et vers l'existence humaine propre ou étrangère. (Hua XLII, p. 247) 
31 une fois, le recours à l'intérieur n'a pas la même valeur selon qu'il est guidé par la quête de la transcendance divine, comme chez saint Augustin, ou par l'élucidation de soi et de son monde. Mais ce parallélisme atteste tout de même une proximité dont la prise en compte ne se réduit pas à un aveu isolé. Ainsi, bien que peu fréquent, ce rapprochement se retrouve lui aussi dans les Conversations avec la sœur Adelgundis Jaegerschmid, sous une forme plus générale qui permet en revanche d'affirmer, non un parallélisme mais une identification : "La philosophie authentique est la même chose que l'auto-réflexion religieuse » (Conversation du 20 avril 1936).

Mais c'est surtout la fin du $\S 35$ de la Krisis qui donne un sens plus précis à cette proximité ou identité, et permet d'y reconnaître autre chose qu'un aveu de circonstance. Husserl y réfléchit au type d'engagement qui se déploie dans l'épochè et qui implique "une certaine attitude professionnelle (eine gewisse berufsartige Einstellung)» (Hua VI, p. 139 ; trad. par G. Granel, p. 154), sans qu'être phénoménologue puisse pour autant valoir comme un métier parmi d'autres : «mettre sur le même pied cette nouvelle science et tous les métiers "bourgeois", et même déjà la mettre sur le même pied que les sciences objectives est une sorte de "bagatellisation (Bagatellisierung)", un mépris de l'immense différence de valeur qu'il peut y avoir d'une façon générale entre les sciences » (Hua VI, p. 139 ; trad. par G. Granel, p. 155). Et c'est précisément pour asseoir la valeur singulière de la pratique "professionnelle " de l'épochè que Husserl recourt (prudemment, il est vrai) au rapprochement avec l'attitude religieuse :

Mais en allant plus loin, cela ne signifie aucunement que l'épochè telle qu'elle a lieu pour le monde de la vie (die lebensweltliche Epoché) [...] ne veut rien dire de plus pour l'existence humaine pratico-«existentielle» que l'époché professionnelle du cordonnier, et que dans le fond cela reviendrait au même que l'on soit cordonnier ou phénoménologue, ou bien même, phénoménologue ou savant dans les sciences positives (positiver Wissenschaftler). Peut-être même se montrera-t-il que l'attitude phénoménologique totale, et l'époché qui en fait partie, sont appelées par essence à produire un changement personnel complet qui serait à comparer en premier avec une conversion religieuse (religiöse Umkehrung), mais qui davantage encore (darüber hinaus) porte en soi la signification de la métamorphose existentielle la plus grande qui soit confiée à l'humanité comme humanité. (Hua VI, p. 140 ; trad. par G. Granel, p. 156)

Comme l'attitude religieuse - et même plus qu'elle -, l'attitude phénoménologique est pour Husserl à même de produire une transformation radicale de l'être humain. Cette transformation a la signification fondamentale d'un renouveau éthique : en ce sens, s'il n'y a pas chez Husserl un "primat de l'éthique » comme plus tard chez Levinas, il y a bel et bien une suprématie de l'éthique, pour autant qu'elle appartient, avec la question religieuse et téléologico-théologique, au niveau suprême vers lequel s'oriente la progression phénoménologique. De même, s'il n'y a certes pas de «tournant théologique » chez Husserl, sa phénoménologie a tout de même esquissé et suivi par différentes voies, comme nous l'avons montré, un «chemin non confessionnel vers Dieu ». Le Dieu de Husserl - ce Dieu susceptible non seulement d'une logification, mais aussi et surtout d'une éthicisation - n'est assurément pas radicalement transcendant, infini ou incompréhensible. Si c'est alors un « Dieu de philosophe », ou un Dieu à portée de l'être humain, croisé et rencontré nécessairement dans l'horizon de l'humain, il ne se réduit pas pour autant à une construction conceptuelle : suscité par l'élucidation transcendantale de la vie humaine, de sa signification et de sa finalité, il est aussi et 
surtout le moteur d'une transformation existentielle où la conversion religieuse et l'épochè dévoilent leur affinitéso.

\section{NOTES}

1. K. Held, "Gott in Edmund Husserls Phänomenologie », in C. Ierna et al. (éds.), Philosophy, Phenomenology, Sciences, Dordrecht, Springer, 2011, p. 723-738, p. 723: "Husserl zu dieser Thematik nichts umfassend Systematisches geschrieben hat, sondern Fragen der metaphysischen Theologie oder der Religionsphilosophie immer nur im Rahmen anderer leitender Problemstellungen aufgeworfen hat». Nous n'avons pas pu consulter, pendant la rédaction de cette Introduction, la thèse de doctorat de son élève Lee Chun Lo, publiée sous le titre Die Gottesauffassung in Husserls Phänomenologie (Francfort-sur-le-Main, Peter Lang, 2008).

2. Ainsi, Jocelyn Benoist notait que « les textes théologiques de Husserl sont rares, disséminés dans les manuscrits de recherche inédits, imprécis et peu convaincants », pour conclure : « II est tentant d'interpréter ainsi les pas gagnés de Husserl vers Dieu comme un pur vagabondage métaphysique " (art. cit., p. 434 et 435 ; voir aussi p. 447). Pour sa part, Emmanuel Housset reconnaît à l'orée de son livre qu' «il n'y a pas d'ouvrage de Husserl consacré à la question de Dieu, ni même une analyse continue dans un texte publié ou dans le Nachlass ", et s'attelle par conséquent à la double tâche d'«établir le dossier historique» et de "produire une interprétation de ce donné textuel » (op. cit., p. 11).

3. L'aveu de Husserl dans ce manuscrit de recherche prend vite un ton nettement plus personnel : " Je ne peux pas mourir sans avoir atteint une clarté relative au sujet des "questions ultimes les plus hautes" »(ibid.).

4. Un peu plus loin dans le même texte, Husserl affirme aussi que «la philosophie (celle qui devient toujours plus concrète) et la théologie (celle qui devient toujours plus philosophique) se recouvrent à l'infini (im Unendlichen decken sich)» (Hua XLII, p. 260).

5. Dans son article de 1991, Jocelyn Benoist soulignait déjà la convergence remarquable qui existe entre ce propos et le manuscrit A VII 9 que nous avons cité plus haut (art. cit., p. 433).

6. Voir aussi, par exemple, dans un cours de 1915 : «Dieu représente l'idée de la connaissance absolue » (Hua XXXVI, p. 93).

7. Dans Husserl et l'idée de Dieu, Emmanuel Housset insiste à plusieurs reprises sur le fait que Husserl déplace radicalement le sens de l'intériorité augustinienne: «pour saint Augustin, l'homme intérieur est celui qui remonte à la source transcendante de toute évidence, alors que pour Husserl, c'est celui qui remonte au principe immanent de tout accomplissement de soi et de toute évidence » (op. cit., p. 177. Voir aussi p. 38 et p. 156).

8. Nous pourrions ainsi parler chez Husserl, en suivant Emmanuel Housset, d'une « religion dans les limites de la simple raison » (op. cit., p. 68).

9. C'est dans cette perspective que Husserl peut affirmer, dans le même texte de 1924, qu' « entre la conscience éthique et la conscience religieuse il n'y a pas de séparation. Le développement éthique se poursuit dans le développement religieux » (Hua XLII, p. 255, n. 1).

10. Parmi d'autres tentatives d'inventorier les voies suivies par Husserl dans son approche de Dieu et de la religion, nous pouvons mentionner celle d'Angela Ales Bello, qui distingue les voies objective, subjective et intersubjective, et parle également d'une voie éthique. Voir, après Husserl sul problema di Dio (Rome, Studium, 1985), Edmund Husserl : pensare Dio, credere in Dio (Padova, 
Messaggero, 2005), traduit en anglais sous le titre The Divine in Husserl and Other Explorations (Dordrecht, Springer, 2009).

11. Notons cependant que dans une lettre à Mahnke de septembre 1917, Husserl avouait son intérêt pour la mystique et les " question de la foi », ainsi que sa résolution de les " approfondir » et de les aborder "par une pensée scientifique dans une pure contemplation " (Briefwechsel, Husserliana Dokumente, vol. III/3, p. 419). Voir également l'analyse de Natalie Depraz dans «Edmund Husserl, Adversus haereses mystikes?», Laval théologique et philosophique, vol. 50, $\mathrm{n}^{\circ}$ 2/1994, p. 327-347.

12. Ce soupçon s'adresse directement à Otto, bien que son ouvrage Le sacré (1917) soit aussi salué comme une contribution à la phénoménologie de la religion. Voir Briefwechsel, Husserliana Dokumente, vol. III/7, p. 205-208 ; trad. par G. Vannier, Les études philosophiques, n 3/1983, p. 349-352.

13. Signalons qu'une édition de cette lettre avant celle de Schuhmann dans la Correspondance, ainsi que sa traduction anglaise accompagnée d'un commentaire, ont été proposées par Herbert Spiegelberg dans un article de 1973 repris dans The Context of the Phenomenological Movement (La Haye, Martinus Nijhoff, 1981).

14. Le corollaire de cette thèse concerne la religion : "Toute religion a ainsi été naïve et par conséquent inintelligible, mais dans l'attitude phénoménologique les thèses naïves de la religion reçoivent non seulement une intelligibilité mais aussi une certaine validité, au même titre que les thèses naïves de notre expérience de la nature ou de n'importe quoi d'autre » (ibid.).

15. La justification de cette affirmation mérite d'être citée elle aussi: «La philosophie phénoménologique, en tant qu'Idée résidant à l'infini, est naturellement "théologie". (Cela veut dire pour moi : la philosophie véritable est eo ipso théologie.) Mais la phénoménologie prise dans l'intervalle temporel, dans la vitalité historique, est dynamis, se trouve en devenir, elle est "méthode", chemin vers la connaissance absolue et universelle, et elle est cette connaissance même en chemin » (Briefwechsel, Husserliana Dokumente III/7, p. 88).

16. Ce passage est cité et traduit par Rudolf Schmitz-Perrin dans son article «Strasbourg, "banlieue de la phénoménologie". Edmond Husserl et l'enjeu de la philosophie religieuse ", in Revue des sciences religieuses, tome 69, fascicule 4, 1995, p. 481-496, p. 492. La lettre à Welch y est présentée comme « un lieu privilégié d'herméneutique permettant de connaître la position du fondateur de la phénoménologie » (ibid.).

17. La persistance de cette problématique d'une monade divine ou d'une conscience omnienglobante s'illustre également dans un texte datant probablement de 1922, édité par Iso Kern au tome XIV des Husserliana (comme Complément XLI) et intitulé par l'éditeur "Possibilité de la fusion des monades. Possibilité d'une sur-monade (divine)» (Hua XIV, p. 300-302). Husserl ne semble avoir tranché cette question difficile du statut d'une conscience ou monade suprême que dans un autre texte, portant le titre "Monadologie ", datant du début des années trente, édité par Iso Kern au tome XV des Husserliana (comme Complément XLVI, p. 608-610) et traduit par Natalie Depraz en annexe de son article «La vie m'est-elle donnée ? Réflexions sur le statut de la vie dans la phénoménologie ", Les études philosophiques, n 4/1991, p. 459-473, p. 466-470 : "Dieu n'est pas lui-même le tout des monades, mais il est l'entéléchie qui est dans ce tout, comme idée d'une évolution téléologique infinie, celle de l'humanité mue par une raison absolue ». Angela Ales Bello, Emmanuel Housset et Klaus Held ont tous les trois analysé ce recours au vocabulaire de l'entéléchie dans leurs travaux déjà cités.

18. Voir notamment le texte $n^{\circ} 11$, datant précisément de 1908 et intitulé «Téléologie, Dieu, possibilité d'une conscience omni-englobante (All-Bewusstsein). Métaphysique fondée dans la phénoménologie transcendantale et phénoménologie ». Cet intitulé indique à lui seul que le levier fondamental de ce prolongement métaphysique de la phénoménologie transcendantale est bien la téléologie. À ce propos, voir la contribution classique de Stephan Strasser, «History, Teleology, and God in the Philosophy of Husserl », Analecta Husserliana, vol. IX, 1979, p. 317-333, 
p. 318: "Contrary to the traditional doctrine God is not, according to him, the "cause" of the world. This is perfectly comprehensible. For Husserl, who had studied astronomy and mathematics, causality was a scientific concept designating a strictly defined function. By contrast, the idea of God was imposed upon him in another context, that of a universal teleology ", à savoir, "the immanent teleology of our conscious life ». Voir aussi, du même auteur, l'étude pionnière « Das Gottesproblem in der Spätphilosophie E. Husserls », Philosophisches Jahrbuch, vol. 67 (1959), p. 130-142.

19. Et Husserl de clore abruptement cette digression : "Cependant, cela ne nous concerne plus ici. Notre visée immédiate n'est pas orientée vers la théologie, mais vers la phénoménologie, si importante que soit la signification que celle-ci présente, indirectement, pour celle-là » (Hua III/ 1, p. 97 ; trad. par J.-F. Lavigne, p. 158).

20. Ce passage, édité désormais au tome XXVII des Husserliana (Hua XXVII, p. 234), a été traduit en français en 1954 par Jean Ladrière et Walter Biemel, à l'occasion de l'étude d'Alwin Diemer déjà mentionnée (art. cit., p. 47-48). Jocelyn Benoist le cite longuement (dans cette même traduction que nous modifions légèrement ici) dans son article de 1991 (art. cit., p. 456-457).

21. Il s'agit du texte $n^{\circ} 14$ du tome XLII. Semblablement, dans un manuscrit de recherche datant probablement des années trente et édité comme texte $n^{\circ} 16$ dans le même volume, nous pouvons lire cette interrogation : «Comment pouvons-nous surmonter le hasard, le destin, la nécessité et la mort - <précisément> dans la volonté de les surmonter, c'est-à-dire dans la croyance [...] que cela ne peut pas nous affecter : que "Dieu est avec nous" ?» (Hua XLII, p. 217).

22. C'est la raison pour laquelle les Leçons sur l'éthique et la théorie des valeurs, publiées au tome XXVIII des Husserliana, contiennent des développements de grande importance pour le sujet qui nous intéresse. Voir par exemple les $\S \S 3$ et 4 du cours de 1911 (Hua XXVIII, p. 175 sqq. en particulier) et le Complément IV, datant de la même année (Hua XXVIII, p. 225 sq.).

23. Comme l'exprime Jocelyn Benoist dans son article déjà cité, selon lequel l'«idolâtrie du concept » ferme la voie à l'expérience de Dieu (art. cit., p. 448-449). Emmanuel Housset décèle lui aussi chez Husserl, dès son article de 1995, un « Dieu de philosophe » (art. cit., p. 482).

24. E. Housset, op. cit., p. 105.

25. Ce qui vaut pour les images de culte vaut aussi pour les textes sacrés et explique sans doute «l'absence de toute référence de Husserl à la Bible ou à des théologiens. Luther lui-même n'est jamais cité » (E. Housset, op. cit., p. 53).

26. Dans son étude « En quête d'une métaphysique phénoménologique : la référence henrienne à Maître Eckhart " (parue dans Michel Henry. L'épreuve de la vie, Actes du colloque de Cerisy 1996, éd. par Alain David et Jean Greisch, Paris, Cerf, La nuit surveillée, 2001, p. 253-279), Natalie Depraz relève également ce passage de la conférence de 1935 sur La crise de l'humanité européenne : " ce n'est que lorsque l'esprit, cessant de se tourner naïvement vers le dehors, revient en lui-même et demeure en lui-même et purement en lui-même, qu'il peut se suffire à lui-même (zu sich selbst zurückkehrt und bei sich selbst und rein bei sich selbst bleibt, kann er sich genügen)» (Hua VI, p. 345-346 ; trad. par G. Granel, p. 380).

27. Voir aussi le témoignage de la lettre à Mahnke du 5 septembre 1917, mentionnée par Rudolf Schmitz-Perrin dans son article déjà cité, où Husserl avoue son intérêt précoce pour la théologie allemande, son attrait et sa curiosité pour la mystique allemande et pour sa «religiosité intérieure (innige Religiosität) » (Briefwechsel, Husserliana Dokumente, vol. III/3, p. 419).

28. Et Husserl d'ajouter : «Voilà qui aidera les théologiens, même si de prime abord il leur semblera qu'il en découle de graves hérésies ».

29. Il s'agit d'un texte qui a d'abord été édité par Schuhmann comme Annexe à une lettre de Husserl à l'Abbé Baudin (Briefwechsel, Husserliana Dokumente, vol. III/7, p. 18-22).

30. Je remercie vivement Natalie Depraz pour ses remarques stimulantes qui m'ont conduite à faire ressortir davantage la portée existentielle et la dimension expérientielle du Dieu husserlien. 